\title{
OROFACIAL GRANULOMATOSIS TREATED WITH SYSTEMIC STEROID: A GASE REPORT
}

\author{
Jha PK
}

Department of Dermatology and Venereology, Institute of Medical Sciences, Banaras Hindu University, Lanka Road Varanasi 221005

\begin{abstract}
Orofacial granulomatosis refers to conditions restricted to oral region mainly characterized by chronic swelling of orofacial tissues with histological evidence of non-caseating granuloma. It may be idiopathic where there is absence of identifiable granulomatous disease or may present as a component of localized or generalized Crohn's disease, tuberculosis and sarcoidosis. The clinical features are facial or lip swelling, angular cheilitis, oral ulcerations, vertical fissures of lips, gingival enlargement, mucosal tags and sometimes lymph node enlargement. A middleaged female presented to our outpatient department with diffuse chronic swelling of lips which after treatment with systemic steroid showed visible improvement during follow up.
\end{abstract}

\section{KEYWORDS}

Orofacial granulomatosis, orofacial swelling, steroid

\section{CORRESPONDING AUTHOR}

Dr. Prasanna Kumar Jha,

Department of Dermatology and Venereology, Institute of Medical Sciences, Banaras Hindu University, Lanka Road Varanasi 221005

Email: jha_prasanna@hotmail.com

Orcid No: https://orcid.org/0000-0001-8475-6611

DOI: https://www.doi.org/10.3126/nmcj.v23i1.36239 


\section{INTRODUCTION}

Orofacialgranulomatosis (OFG) refers to chronic swelling of orofacial tissues with histological evidence of noncaseating granuloma. ${ }^{1}$ Idiopathic orofacial granulomatosis refers to conditions restricted to oral region without any identifiable systemic granulomatous diseases. The term orofacial granulomatosis was proposed by Weisenfield et al in 1985 as a description for non-infectious granulomatous disorders of the lips, face and oral cavity that are histologically associated with noncaseating epithelioid granulomas and multinucleated Langhans (foreign body) type giant cells within the oral mucosa. ${ }^{1}$ It may be idiopathic or may present as a component of localized or generalized Crohn's disease, tuberculosis, actinomycosis and sarcoidosis. ${ }^{2}$ The clinical features are facial or lip swelling, angular cheilitis, oral ulcerations, vertical fissures of lips, gingival enlargement, mucosal tags and sometimes lymph node enlargement. OFG may be the indication of certain conditions like sarcoidosis, Inflammatory bowel disease, granulomatosis with polyangiitis (GPA), Melkersson-Rosenthal syndrome (MRS). ${ }^{3,4}$ Idiopathic orofacial granulomatosis has been linked to contact hypersensitivity with dietary factors and elements like cobalt. ${ }^{5-7}$

\section{CASE REPORT}

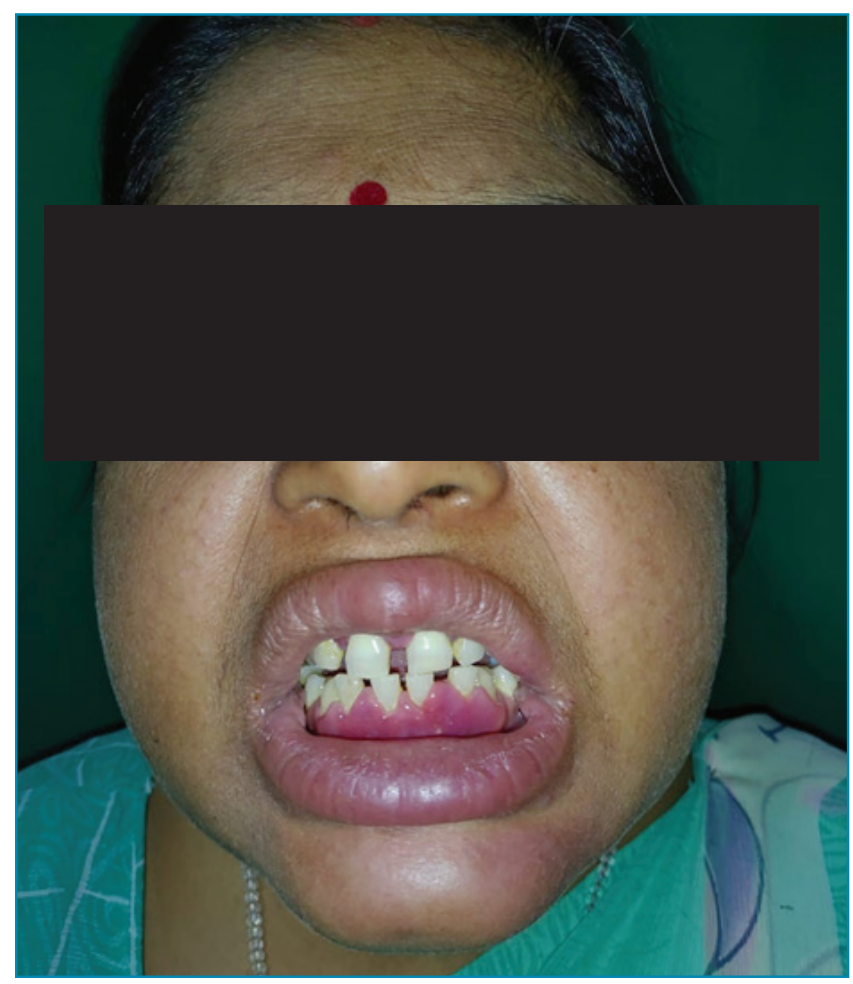

Fig. 1: An erythematous, scaly plaque of size $5 \mathrm{~cm} \times 2 \mathrm{~cm}$ was present over chin.
A 40-year-old female, presented in Banaras Hindu University, Varanasi Dermatology outpatient department with diffuse, soft, painless enlargement of lips for six months. Mild fissures were present on the upper and lower lips. The patient had gingival enlargement in

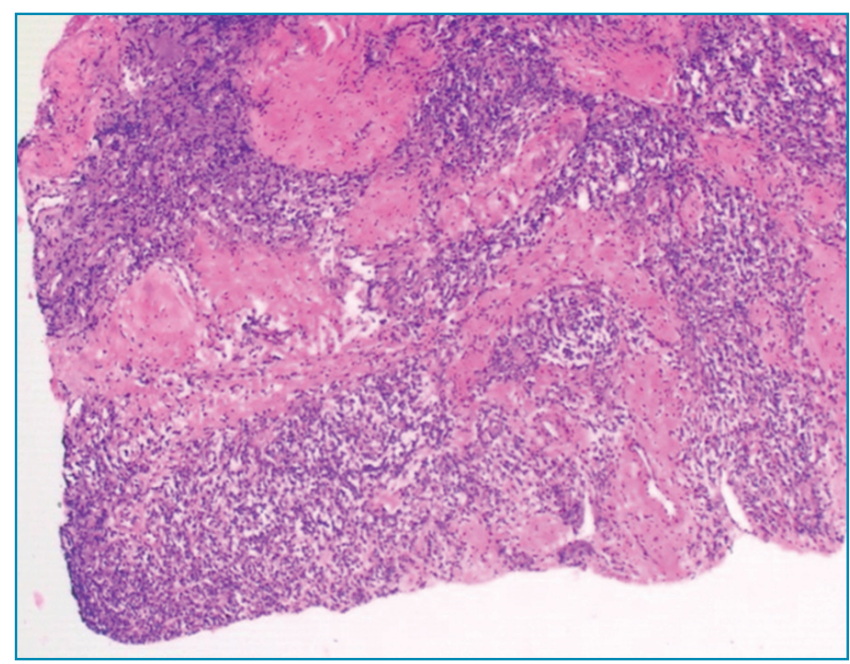

Fig. 2: Microphotograph of skin biopsy showing dense, diffuse, infiltrate of lymphocytes, histiocytes and plasma cells in a patchy pattern throughout the submucosa. There was no well-defined granuloma. Overlying epidermis showed mild focal spongiosis and slight hyperplasia. The histopathology was suggestive of orofacial granulomatosis (OFG). [H\&E stain, 400x]

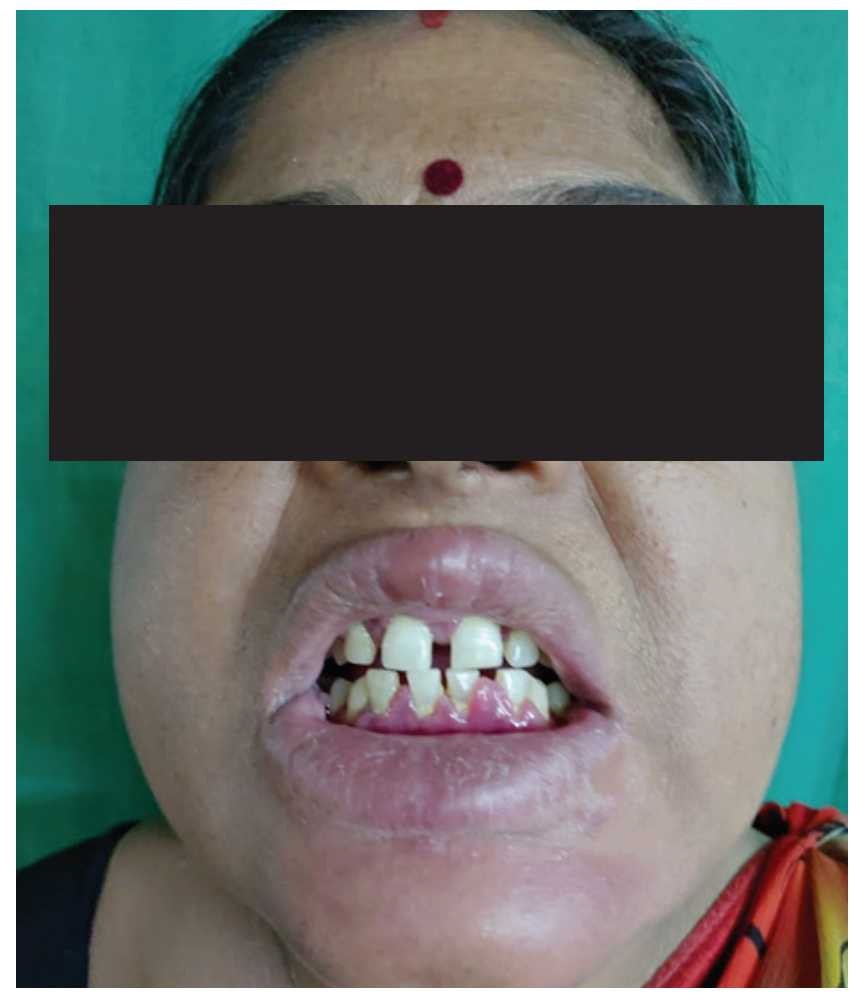

Fig. 3: After one month, the patient showed visible improvement. 
the upper and lower arch. This enlargement involved attached, interdental gingiva and covered almost one-third to one-half of the crown length reaching mucogingival junction in the maxilla and mandible. An erythematous, scaly plaque of size $5 \mathrm{~cm} \mathrm{X} 2 \mathrm{~cm}$ was present over chin (Fig. 1). We kept a differential diagnosis of cheilitis granulomatosa, sarcoidosis, cutaneous tuberculosis and oro-facial granulomatosis. Routine blood investigations, chest X-Ray, Mantoux test, stool occult blood, colonoscopy, serum angiotensin converting enzyme (ACE) levels revealed no abnormality. An incisional biopsy of the mandibular gingiva was performed which on histopathology showed dense, diffuse, infiltrate of lymphocytes, histiocytes and plasma cells in a patchy pattern throughout the submucosa. Non caseating granuloma was present but it was poorly defined. Overlying epidermis showed mild focal spongiosis and slight hyperplasia. The histopathology was suggestive of OFG (Fig. 2).

Our patient refused for intralesional triamcinolone injection, so we started her on Prednisolone $40 \mathrm{mg} /$ day with tapering by 10 mg every ten days. After one month, the patient showed visible improvement (Fig. 3). After one month, the patient lost to follow up.

\section{DISCUSSION}

Orofacial granulomatosis is a chronic swelling of orofacial tissues which can be a major cosmetic concern for the patient but also its association with systemic diseases which needs to be looked out and screened for during initial visit as well as follow up. The treatment of OFG is difficult, particularly in the absence of an etiologic factor during initial visit. Although rare, spontaneous remission is possible. Management includes excluding dietary allergens and materials containing cobalt in few case reports..$^{5-7}$ The various treatment modalities which have been tried include intralesional steroid injections, systemic steroids and surgical excision. Clofazimine has been reported to be effective in the management of OFG. ${ }^{8}$ Low-dose thalidomide has been shown to be successful in treating OFG. ${ }^{9}$ In recent years, infliximab, a chimeric monoclonal antibody against TNF-a has shown efficacy. ${ }^{10}$

Most of the cases of OFG has been linked to systemic involvement, which sounds the need for proper evaluation to rule out inflammatory bowel disease, tuberculosis or any other systemic illness, early diagnosis of which can lead to better prognosis. ${ }^{2}$

\section{REFERENCES}

1. Wiesenfeld D, Ferguson MM, Mitchell DN et al. Oro-facial granulomatosis-a clinical and pathological analysis. Q J Med 1985; 54: 101-13.

2. Abha PR. Orofacial granulomatosis: A case report with review of literature. J Indian Soc Periodontal 2012; 16: 469-74.

3. Girlich C, Bogenrieder T, Palitzsch KD et al. Orofacial granulomatosis as initial manifestation of Crohn's disease: a report of two cases. Eur J Gastroenterol Hepatol 2002; 14: 873-6.

4. Mignogna MD, Fedele S, Lo Russo L et al. Orofacial granulomatosis with gingival onset. $J$ Clin Periodontol 2001; 28: 692-6.

5. Patton DW, Ferguson MM, Forsyth A, James J. Oro-facial granulomatosis: A possible allergic basis. Br J Oral Maxillofac Surg 1985; 23: 235-42.

6. Pryce DW, King CM. Orofacial granulomatosis associated with delayed hypersensitivity to cobalt. Clin Exp Dermatol 1990; 15: 384-6.
7. Reed BE, Barrett AP, Katelaris C, Bilous M. Orofacial sensitivity reactions and the role of dietary components. Case reports. Aust Dent J 1993; 38: 287-91.

8. Sussman GL, Yang WH, Steinberg S. MelkerssonRosenthal syndrome: clinical, pathologic, and therapeutic considerations. Ann Allergy 1992; 69: 187-94.

9. Hegarty A, Hodgson T, Porter S. Thalidomide for the treatment of recalcitrant oral Crohn's disease and orofacial granulomatosis. Oral Surg Oral Med Oral Pathol Oral Radiol Endod 2003; 95 : 576-85.

10. Peitsch WK, Kemmler N, Goerdt S, Goebeler M. Infliximab: A Novel Treatment Option for Refractory Orofacial Granulomatosis. Acta Derm Venereol 2007; 87: 265-6. 